\title{
Characterization of Shape of Polymer Nano-Films Possessing Various Crosslinking Chain Length
}

\author{
Shin-ichi Kondo ${ }^{1 *}$, Naoki Doi ${ }^{1}$, Yasushi Sasai ${ }^{2}$, Yukinori Yamauchi ${ }^{3}$, \\ and Masayuki Kuzuya ${ }^{4}$ \\ ${ }^{1}$ Laboratory of Pharmaceutical Physical Chemistry, Gifu Pharmaceutical University, \\ 1-25-4 Daigaku-Nishi, Gifu 501-1196, Japan \\ ${ }^{2}$ Faculty of Pharmacy, Gifu University of Medical Science, 4-3-3 Nijigaoka, Kani-shi, \\ Gifu 509-0293, Japan \\ ${ }^{3}$ Department of Pharmaceutical Physical Chemistry, College of Pharmaceutical Sciences, \\ Matsuyama University, 4-2 Bunkyo-cho, Matsuyama, Ehime 790-8578, Japan \\ ${ }^{4}$ Department of Health and Welfare, Faculty of Human Welfare, Chubu Gakuin \\ University, 2-1 Kirigaoka, Seki-shi, Gifu 501-3993, Japan \\ *skondo@gifu-pu.ac.jp
}

\begin{abstract}
In this paper we studied on the size and morphology of polymer nano-film synthesized with the linker possessing the various length. Although the size measured by Zetasizer was larger than that by AFM, it was considered that polymer nano-film might be expanded due to solvation in PBS. The morphology of the polymer nano-film synthesized from succinic acid and glutaric acid showed square morphology rather than sphere. On the other hand, the polymer nano-film from azelaic acid showed spherical morphology. It was suggested that the difference among them might be ascribe to the flexibility of a polymer nano-film. Thus, the longer crosslinking chain length could be a more flexible polymer nano-film.
\end{abstract}

Keywords: Polymer nano-film, Crosslinking chain length, Plasma irradiation, Self-assembled phospholipid layer, Cyclodextrin

\section{Introduction}

The nano-film (or nano-sheet) is a new type of material that possesses a two-dimensional polymeric structure with nano-meter thickness. Many of nano-film needs the supporting material [1-5]. Recently, free standing nano-films fabricated from molecular, atomic, and ionic components have been extensively investigated for systems with analytical and biomedical applications, such as separation matrices and drug delivery carriers [6-11].

We have developed the method to introduce a durable surface wettability on several hydrophobic polymers by plasma-assisted method [12-16], and to fabricate a self-assembled phospholipid (phosphatidyl choline (PC)) layer (LDPE-PC-SA) on it [17]. It was also confirmed that LDPE-PC-SA possessed fluidity resembling to cellular membrane.

In recent years we have developed the polymer nano-film possessing hydrophilic and hydrophobic surface by using LDPE-PC-SA containing stearic acid (StA) (Fig. 1). The morphology of polymer nano-film in organic solvents (methanol and chloroform) and water was estimated by ${ }^{1} \mathrm{H}-\mathrm{NMR}$ spectra measurement and atomic force microscope (AFM) measurement [18-20].

It was assumed that the length of linker moiety might affect on the size and morphology of the polymer nano-film. In this paper we studied on the size and morphology of polymer nano-film synthesized with the linker possessing the various length according to the previous method $[18,19]$. We selected three kinds of linker shown in Fig. 2, succinic acid, glutaric acid and azelaic acid. The particle size and distribution of polymer nano-film in phosphate buffer solution was estimated by using Zeta sizer by dynamic light scattering. The morphology of polymer nano-film in dry state was also estimated by atomic force microscope (AFM).

$\begin{array}{lll}\text { Received } & \text { April } & 1,2021 \\ \text { Accepted } & \text { May } & 3,2021\end{array}$




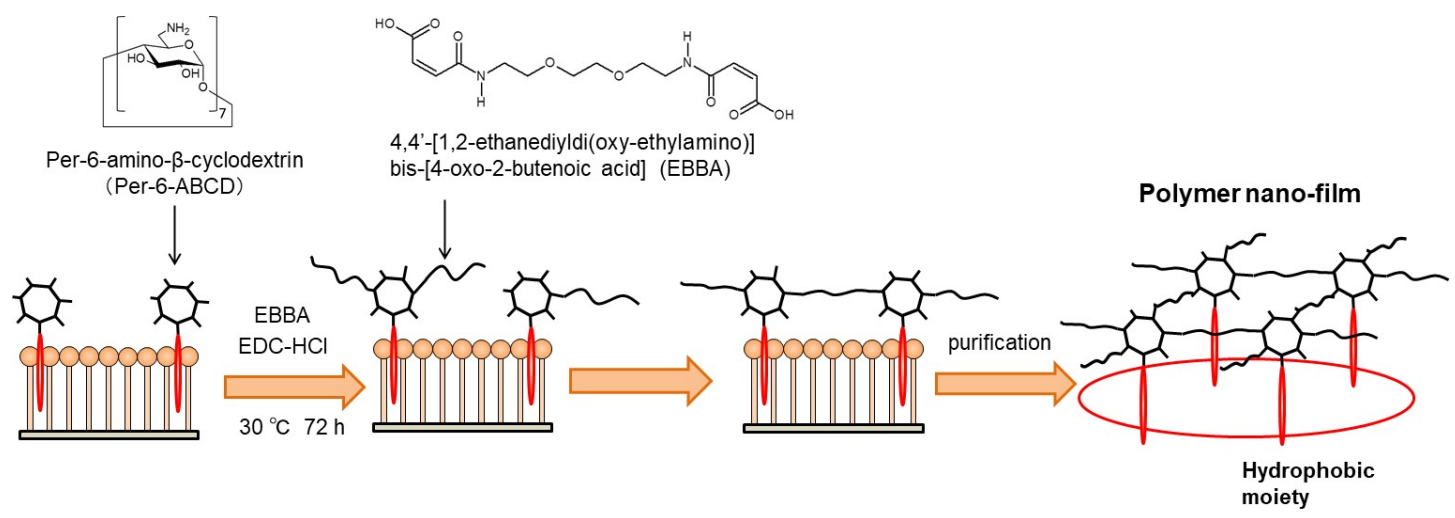

Fig. 1. Schematic illustration of the fabrication of polymer nano-film possessing hydrophilic and hydrophobic side with the self-assembled phospholipid layer containing stearic acid.

$$
\begin{aligned}
\mathrm{HOOC}-\left(\mathrm{CH}_{2}\right)_{\mathrm{n}}-\mathrm{COOH} \\
\mathrm{n}=2 ; \text { succinic acid } \\
\mathrm{n}=3 ; \text { glutaric acid } \\
\mathrm{n}=7 ; \text { azelaic acid }
\end{aligned}
$$

Fig. 2. Structures of linker moiety (dicarboxylic acid) which replaced EBBA shown in Fig. 1.

\section{Experimental}

\subsection{Materials}

Succinic acid was purchased from Kishida Chemical Co., Ltd. Glutaric acid and azelaic acid were obtained from Nakalai Tesque Inc. Per-6amino- $\beta$-cyclodextrin (Per-6-ABCD) was synthesized according to the literatures [21].

\subsection{Preparation of polymer nano-film}

According to the literatures, the self-assembled phospholipid layer incorporating stearic acid (StA) (LDPE-StA-PC-SA) was fabricated, and then Per-6-ABCD was immobilized on the LDPE-StAPC-SA film $[17,18]$. To $100 \mathrm{~mL}$ of water was added $1 \mathrm{~mL}$ of $0.047 \mu \mathrm{mol} / \mathrm{ml}$ 1-ethyl-3-(3dimethylaminopropyl) carbodiimide hydrochloride $(\mathrm{EDC}-\mathrm{HCl})$ water solution and $50 \mu \mathrm{L}$ of 29 $\mu \mathrm{mol} / \mathrm{ml}$ succinic acid in water. This solution was kept at $30{ }^{\circ} \mathrm{C}$ for $30 \mathrm{~min}$. The LDPE-StA-PC-SA film immobilizing Per-6-ABCD $(1 \times 3 \mathrm{~cm}, 40$ films) was soaked into this solution at $30{ }^{\circ} \mathrm{C}$ for 18 h. These films were washed with water. The films were immersed into $60 \mathrm{~mL}$ of ethanol and kept at $30{ }^{\circ} \mathrm{C}$ for $3 \mathrm{~h}$. This ethanol solution was concentrated to about $7 \mathrm{~mL}$ in vacuo. The concentrated solution was transferred into a pre-swollen semi-permeable membrane (Spectra/ Por® 1 Dialysis Membrane Standard RC Tubing
MWCO: 6 - $8 \mathrm{kD}$, Spectrum Laboratories, Inc.). The both sides of the tube were sealed with dialysis tubing closures (Dialysis Tubing Closures Standard Closure Type, $80 \mathrm{~mm}$ ). The solution was dialyzed against $300 \mathrm{~mL}$ of methanol for $15 \mathrm{~h}$. And then the dialysis membrane was immersed into $300 \mathrm{~mL}$ of pH 7.4 phosphate buffer saline (PBS) to obtain the polymer nano-film solution. The polymer nano-films crosslinked with glutaric acid or azelaic acid were also prepared in the similar manner.

\subsection{Size analysis and particle distribution}

The particle size (hydrodynamic radius and mean-square radius of gyration) and distribution of polymer nano-film were determined by using Zeta sizer by dynamic light scattering (Malvern Zetasizer nano ZS, Malvern instruments, UK). Three replicates were measured.

2.4 Atomic force microscope measurement in dynamic force mode

The PBS solution of polymer nano-film was exchanged into distilled water. The water solution of polymer nano-film was casted on mica, and then dried to obtain AFM sample. The morphology of polymer nano-film was investigated by AFM with dynamic force mode employing Scanning Probe Microscopy AFM-5400L (Hitachi HighTechnologies Co.) and cantilever (MPP-11100-10, Veeco). AFM measurement was performed under atmospheric conditions.

\section{Results and Discussion}

3.1. Particle size of polymer nano-film in PBS The particle size and distribution of polymer nano-film was measured by Zetasizer in PBS. Figure 3 shows the number average particle diameter and size distribution of polymer nano-film synthesized with various linker (dicarboxylic acid). 
The character in the parentheses denotes the number of methylene carbon in the linker. The particle diameter of polymer nano-film synthesized from succinic acid was smallest among them, and that from glutaric acid was largest. The size distribution of the polymer nano-film from succinic acid was narrower than the others. The $\rho$ value was represented by the ration of the mean-square radius of gyration $\left(<S^{2}>\right)$ and the hydrodynamic radius $\left(R_{\mathrm{h}}\right), \rho=\left\langle S^{2}>/ R_{\mathrm{h}}\right.$, and used to estimate the morphology of polymer in a solution. The $\rho$ values of polymer nano-film from succinic acid and azelaic acid were about 1.3 and 0.5 , respectively. When the $\rho$ value was less than 1 , the morphology of polymer was estimated as a shrink form such as spherical structure. On the other hand, in the case of more than 1 the morphology was assumed as an expanded form and the gaussian chain was expected in the case of 1.5. Therefore, it was suggested that the polymer nano-film from succinic acid might be an expanded morphology like a film and that the structure in the case of azelaic acid might be spherical.

\subsection{Morphology estimation of polymer nano-film} in dry condition by AFM measurement

The water solution of polymer nano-film was casted on mica, and then dried to obtain the AFM sample. Figure 4 shows the AFM images of polymer nano-film synthesized form three kinds of linkers.

Although the number average particle diameter estimated on AFM measurement was clearly smaller than those on Zetasizer, the order of average diameter from AFM measurement was coincident with those from Zetasizer. It was considered that polymer nano-film might be expanded due to solvation in PBS. The morphology of polymer nano-film from succinic acid showed nearly rectangle shape, and the average height was lowest among them. It was presumed that the shorter linker length might cause the steric hindrance between Per-6-ABCDs under crosslinking process, so that the smaller polymer nano-film could be formed. As described above it was assumed that the morphology of this polymer nano-film could be an expanded form from the $\rho$ value. As shown in Fig. 4(B) the polymer nano-film synthesized from glutaric acid demonstrated square morphology rather than sphere and the largest size among them. On the other hand the polymer nano-film synthesized from azelaic acid showed spherical morphology and its size was smaller than that from glutaric acid. It was considered from these results that glutaric acid might produce a larger polymer nano-film than that from succinic acid due to longer linker. As the polymer nano-film of azelaic acid had a long alkyl group, it was expected that this nano-film would possess high flexibility. Therefore, it was considered that this polymer nano-film could show the spherical morphology having hydrophobic surface inside. The similar result was obtained from the $\rho$ value. We had reported that the polymer nano-film possessing longer linker chain than azelaic acid showed spherical morphology by observing AFM measurement [19].

\section{Conclusion}

The conclusions drawn from the present study can be summarized as follows.

Although the size measured by Zetasizer was larger than that by AFM, it was considered that polymer nano-film might be expanded due to solvation in PBS. The morphology of the polymer nano-film synthesized from succinic acid and glutaric acid showed square morphology rather than sphere. On the other hand, the polymer nano-film from azelaic acid showed spherical morphology. It was suggested that the difference among them might be

(A) succinic acid (C2)

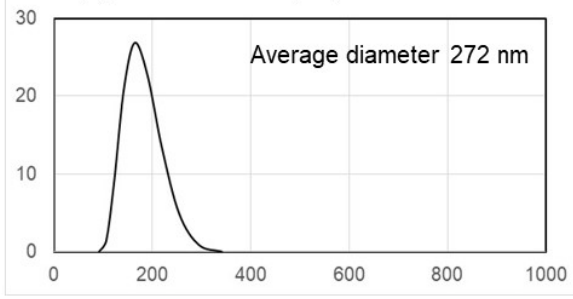

(B) glutaric acid (C3)

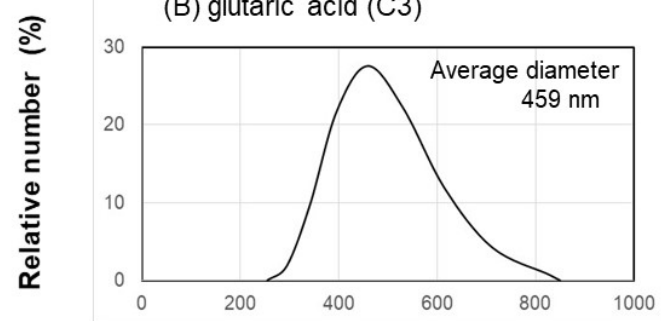

(C) azelaic acid (C7)

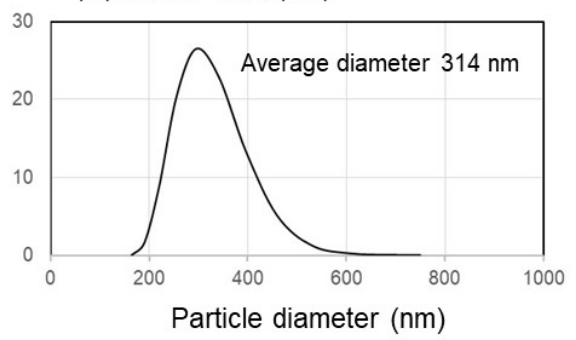

Fig. 3. Number average particle diameter and size distribution of polymer nano-film synthesized form (A) succinic acid, (B) glutaric acid, and (C) azelaic acid. 
(A) succinic acid (C2)

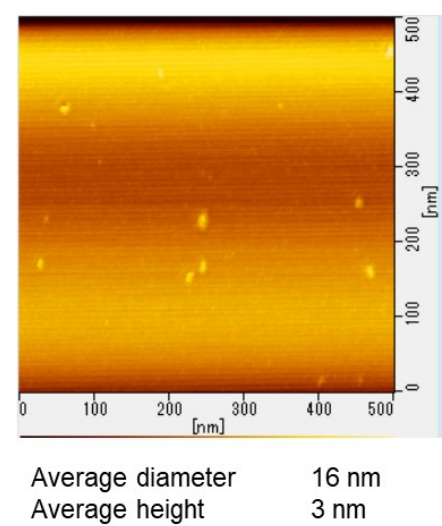

(B) glutaric acid (C3)

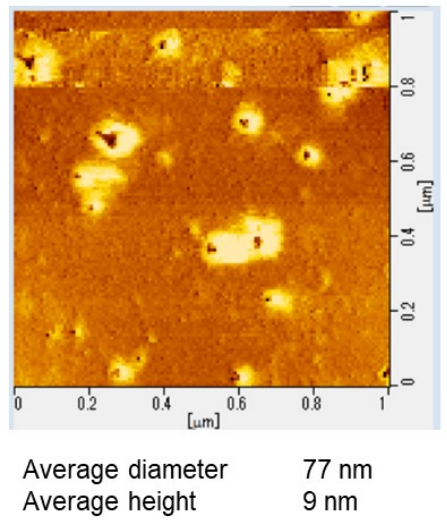

(C) azelaic acid (C7)

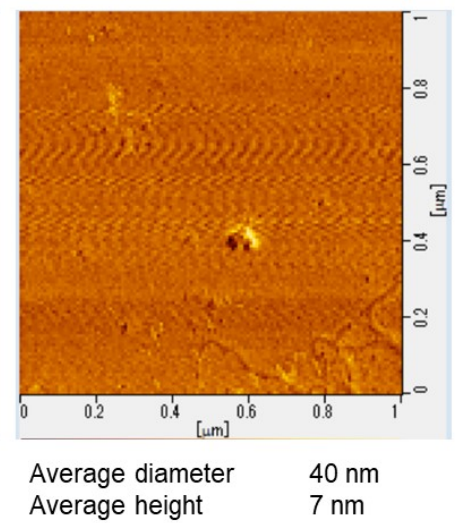

Fig. 4. AFM images of polymer nano-film synthesized form (A) succinic acid, (B) glutaric acid, and (C) azelaic acid.

ascribe to the flexibility of a polymer nano-film. Thus, the longer crosslinking chain length could be a more flexible polymer nano-film.

We are now actively elaborating the application to a drug carrier possessing more effective drug release property using polymer nano-film.

\section{Acknowledgment}

This work was financially supported in part by the OGAWA Science and Technology Fundation, which is gratefully acknowledged.

\section{References}

1. H. Tanak, M Mitsuishi, and T. Miyashita, Chem. Lett., 34 (2005) 1246.

2. H. Endo, Y. Kado, M. Mitsuishi, and T. Miyashita, Macromolecules, 39 (2006) 5559.

3. H. Zhu, M. Mitsuishi, and T. Miyashita, Macromolecules, 45 (2012) 9076.

4. K. Takada, R. Sakamoto, S.-T. Yi, S. Katagiri, T. Kambe and H. Nishihara, J. Am. Chem. Soc., 137 (2015) 4681.

5. C. Kuru, C. Choi, A. Kargar, D. Choi, Y. J. Kim, C. H. Liu, S. Yzvuz, and S. Jin, Adv. Sci., 2 (2015) 1500004.

6. T. Bauer, Z. Zheng, A. Renn, R. Enning, A. Stemmer, J. Sakamoto, and A. D. Schlüter, Angew. Chem., 50 (2011) 7879.

7. S. Motoyama, R. Makiura, O. Sakata, and H. Kitagawa, J. Am. Chem. Soc., 133 (2011) 5640.

8. Z. Zheng, L. Opilik, F. Schiffmann, W. Liu, G. Bergamini, P. Ceroni, L.-T. Lee, A. Schlütz, J. Sakamoto, R. Zenobi, J. VandeBondele, and A. D. Schlüter, J. Am. Chem. Soc., 136 (2014) 6103.
9. B. Ni, M. Huang, Z. Chen, Y. Chen, C.-H. Hsu, Y. Li, D. Pochan, W.-B. Zhang, S. Z. D. Cheng, and X.-H. Dong, J. Am. Chem. Soc., 137 (2015) 1392.

10.N. Idota, S. Fukuda, T. Tsukahara, and Y. Sugahara, Chem. Lett., 44 (2015) 203.

11. B. P. Nsdyskoyi, Y. Li, M. Imura, N. Miyamoto, T. Nakato, T. Sasaki, and Y. Yamauchi, Angew. Chem., 54 (2015) 4222.

12.M. Kuzuya and T. Yamashiro, J. Photopolym. Sci. Technol., 8 (1995) 381.

13.M. Kuzuya, Y. Matsuno, T. Yamashiro, and M. Tuiki, Plasmas Polym., 2 (1997) 79.

14.M. Kuzuya, T. Yamashiro, S. Kondo, and M. Tuiki, Plasmas Polym., 2 (1997) 133.

15.M. Kuzuya, T. Sawa, T. Yamashiro, S. Kondo, and O. Takai, J. Photopolym. Sci. Technol., 14 (2001) 87.

16.S. Kondo, Y. Sasai, and M. Kuzuya, Thin Solid Films, 515 (2007) 4136.

17.S. Kondo, Y. Sasai, Y. Yamauchi, and M. Kuzuya, J. Photopolym. Sci. Technol., 22 (2009) 477.

18.S. Kondo, T. Kusumoto, Y. Sasai, N. Doi, Y. Yamauchi, and M. Kuzuya, J. Photopolym. Sci. Technol., 29 (2016) 439.

19.S. Kondo, Y. Sasai, Y. Yamauchi, and M. Kuzuya, J. Photopolym. Sci. Technol., 31 (2018) 385.

20.S. Kondo, Y. Sasai, Y. Yamauchi, and M. Kuzuya, J. Photopolym. Sci. Technol., 32 (2019) 541.

21.S. Kondo, M. Suzuki, Y. Sasai, Y. Yamauchi, and M. Kuzuya, J. Photopolym. Sci. Technol., 26 (2013) 545 . 